\title{
Phenyliodine(III) diacetate as a mild oxidant for aziridination of olefins and imination of sulfoxides with $N$-aminophthalimide
}

\author{
Larisa B. Krasnova, Ryan M. Hili, Olga V. Chernoloz, and Andrei K. Yudin* \\ Davenport Research Laboratories, University of Toronto, Department of Chemistry, 80 St. \\ George Street, Toronto, Ontario, M5S 3 H6 \\ E-mail: ayudin@,chem.utoronto.ca
}

\section{Dedicated to Professor N.S. Zefirov on the occasion of his $70^{\text {th }}$ birthday}

(received 29 Sept 04; accepted 10 Dec 04; published on the web 18 Dec 04)

\begin{abstract}
Phenyliodine(III) diacetate (PIDA) was found to promote facile nitrene transfer to olefins and sulfoxides giving aziridines and sulfoximines, respectively, in high isolated yields and with high selectivities. The reactions are tolerant for a range of functional groups and proceed under mild conditions. The feasibility of scale-up has been demonstrated.
\end{abstract}

Keywords: Aziridination, sulfoximination, $N$-aminophthalimide, aziridines, sulfoximines, oxidation

\section{Introduction}

Due to frequent occurrence of nitrogen atoms in the structures of biologically active compounds, chemoselective functionalization of organic molecules with nitrogen-containing groups is among the most important goals in organic synthesis. Recently, a great progress in this field has been achieved. Many amination protocols have been developed and their utilization in organic synthesis has been demonstrated. ${ }^{1}$ Our group recently reported electrochemical aziridination ${ }^{2 a}$ of olefins and imination of sulfoxides with $N$-aminophthalimide. ${ }^{2 b} 1$-Phthalimidoaziridines can also be obtained using lead tetraacetate(IV) (LTA) as an oxidant. ${ }^{3}$ Unfortunately, application of LTA is limited due to its high toxicity and difficulties associated with storage and handling. ${ }^{4}$ Thus, introduction of an alternative reagent, which would be stable, non-toxic (unlike LTA) and readily available (unlike electrochemical equipment) is of great interest. Herein we report aziridination of olefins and sulfoximination of sulfoxides with $\mathrm{N}$-aminophthalimide and phenyliodine(III) diacetate (PIDA) as a mild oxidant. ${ }^{5}$ 


\section{Results and Discussion}

The mechanism of olefin aziridination with $N$-aminophthalimide $\mathbf{1}$ has been studied by Atkinson and coworkers. ${ }^{6}$ It is believed that $\mathrm{N}$-acetoxyaminophthalimide 2 , which is generated upon oxidation of $\mathrm{N}$-aminophthalimide $\mathbf{1}$ in the presence of acetate ion, is the reactive intermediate during aziridine 3 formation (Scheme 1). In the absence of any trapping nucleophile or in the case when the nucleophile is not reactive enough, $\mathbf{2}$ reacts with $\mathbf{1}$ with the formation of tetrazane $\mathbf{4}$, which is further oxidized to tetrazene $\mathbf{5}$. The former ultimately decomposes to phthalimide 6 .

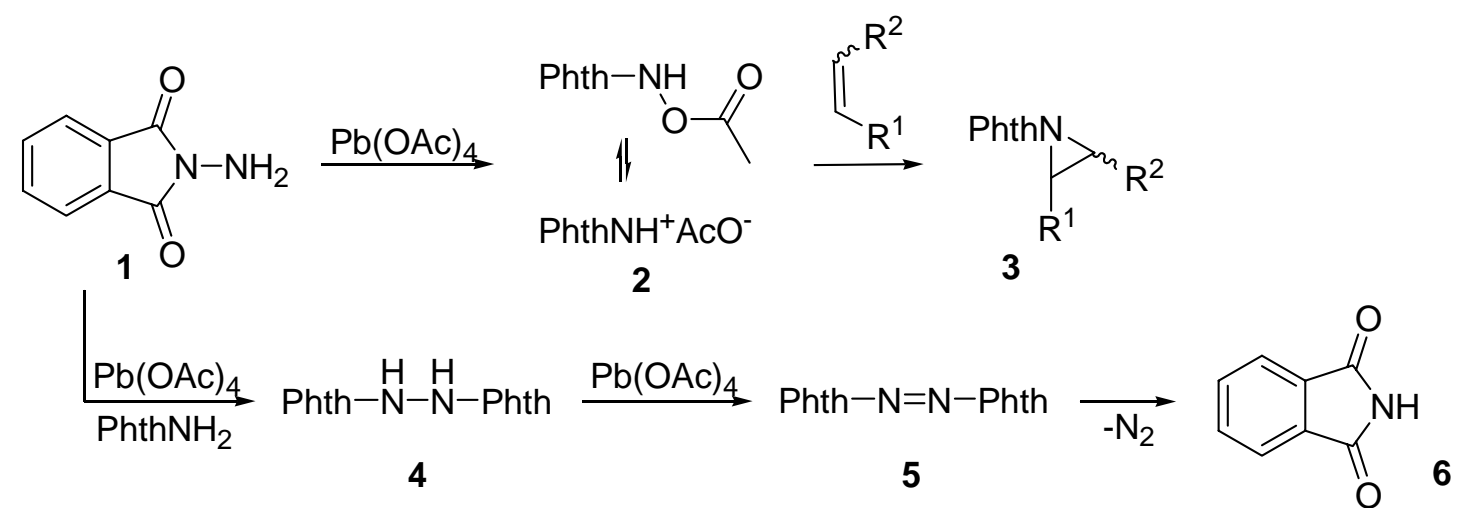

\section{Scheme 1}

Understanding the nature of the reactive intermediate and mechanism of the nitrene transfer led us to the assumption that a mild electrophilic oxidant such as PIDA can serve as an oxidant in the aziridination reaction. As in the case of phenol oxidation with PIDA, ${ }^{7}$ the mechanism of generating the $\mathrm{N}$-acetoxy nitrenium ion from $\mathrm{N}$-aminophthalimide can be explained according to Scheme 2. The $N$-aminophthalimide is oxidized by PIDA producing intermediate 7 . The phenyliodide group dissociates from 7 with the formation of the acetoxy nitrenium ion $\mathrm{N}$ acetoxyaminophthalimide 2 , the reactive intermediate of the olefin aziridination. The byproducts of this process are acetic acid and iodobenzene. In the case of certain olefins containing electron-withdrawing groups (such as dimethyl maleate and maleic acid anhydride) that do not react under these conditions or give products in small yields, substantial amounts of tetrazane 4 and tetrazene 5 were isolated.

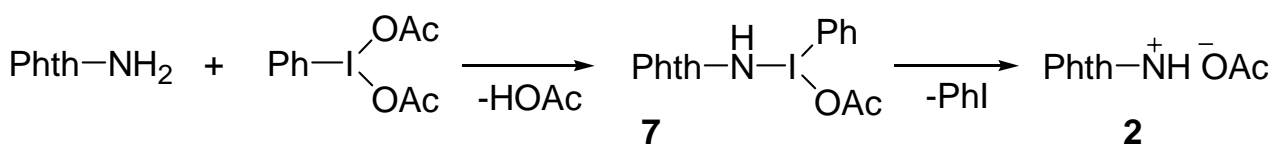

\section{Scheme 2}


The reaction conditions were optimized for cyclohexene as a model substrate. Dichloromethane was found to be the best solvent (Table 1. Optimization of the reaction conditions, entry 4). In a typical run, $\mathrm{PhthNH}_{2}$ gradually dissolves after addition of the oxidant within 2 minutes in $\mathrm{CHCl}_{3}, 20-40$ minutes in $\mathrm{CH}_{2} \mathrm{Cl}_{2}$, and 30 minutes in $\mathrm{CH}_{3} \mathrm{CN}$. The reaction is complete within 2 hours in $\mathrm{CH}_{2} \mathrm{Cl}_{2}$ (monitored by TLC), and after basic work-up aziridines were purified by column chromatography. At the end of the reaction, precipitation of the tetrazane and tetrazene by-products may be observed, depending on the solvent used and the nature of the olefin, which indicates the reduced yield of the product (unless the formed aziridine has a low solubility).

Table 1. Optimization of the reaction conditions

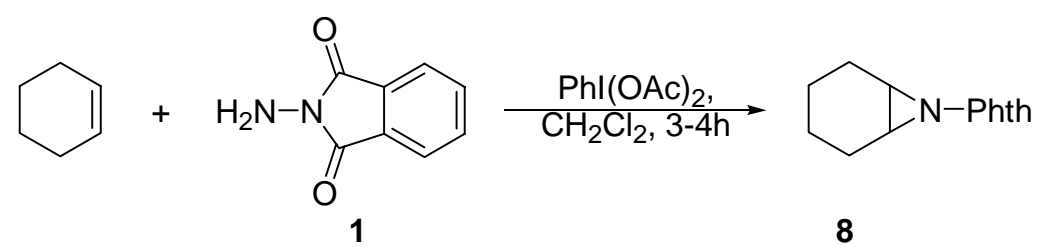

\begin{tabular}{cccc}
\hline Entry & Solvent & Equivalents of PIDA/PhthNH & Yield, \%* \\
\hline $\mathbf{1}$ & $\mathrm{CH}_{2} \mathrm{Cl}_{2}$ & $1: 1$ & 31 \\
$\mathbf{2}$ & & $1: 1.2$ & 44 \\
$\mathbf{3}$ & & $1: 1.5$ & 59 \\
$\mathbf{4}$ & & $1.5: 2$ & 78 \\
$\mathbf{5}$ & $\mathrm{CHCl}_{3}$ & $1.5: 1$ & 43 \\
$\mathbf{6}$ & & $1.5: 2$ & 73 \\
$\mathbf{7}$ & $\mathrm{CH}_{3} \mathrm{CN}$ & $1.5: 1$ & 36 \\
\hline
\end{tabular}

*Procedure: PIDA $(0.5 \mathrm{mmol}-0.75 \mathrm{mmol})$ was dissolved in $4 \mathrm{ml}$ solvent and gradually added to the mixture of $0.5 \mathrm{mmol}$ olefin and $\mathrm{PhthNH}_{2}(0.5 \mathrm{mmol}-1 \mathrm{mmol})$ in $1 \mathrm{ml}$ solvent. After $3-4 \mathrm{~h}$ the reaction mixture was quenched with $5 \mathrm{ml}$ saturated $\mathrm{NaHCO}_{3}$ and worked up.

The optimized reaction conditions were applied to a variety of olefins. Aliphatic olefins usually give good yields (Table 2, entry 1-3). The reaction tolerates a variety of functional groups such as an ester, a carbonyl function, a nitrile, a hydroxyl group and an amide (Table 2, entry 5-10), although the yield for the olefins with a primary hydroxyl group was found to be lower than for the acetylated ones (Table 2, entry 6,7). Some of the $N$-phthalimidoaziridines exist as mixtures of invertomers, which have distinctly different ${ }^{1} \mathrm{H}$ and ${ }^{13} \mathrm{C}$ NMR signals. 
Table 2. Substrate scope: functional group compatibility

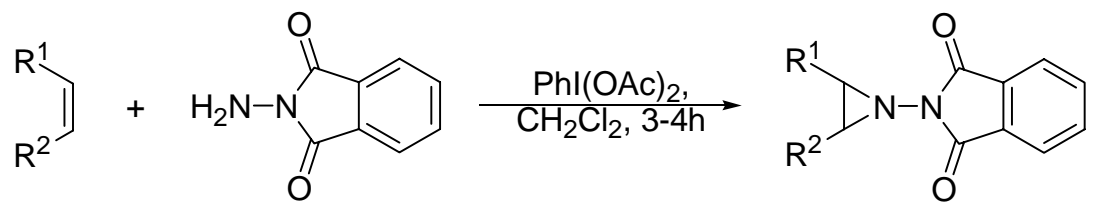

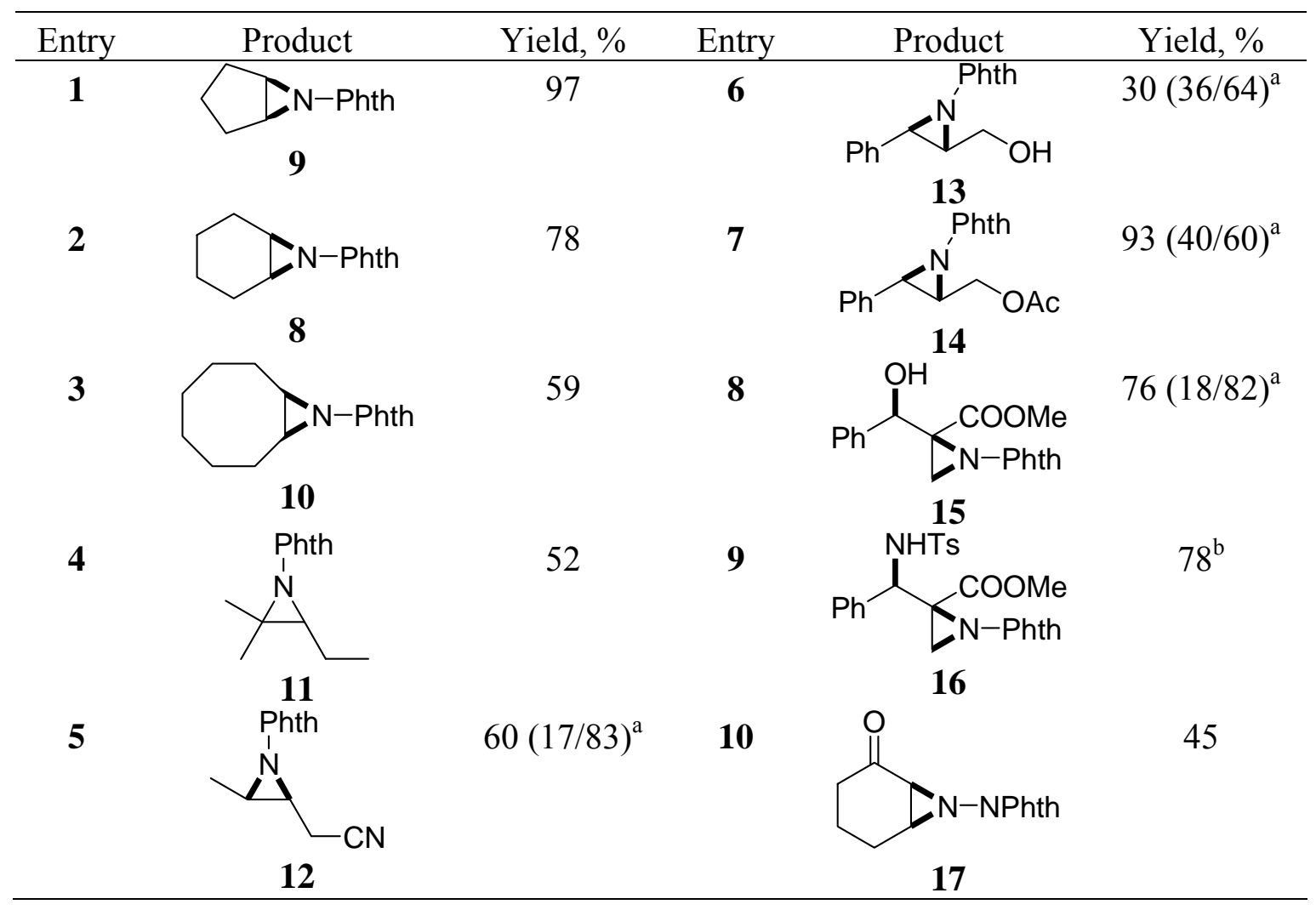

${ }^{\mathrm{a}}$ Ratio of invertomers was determined by ${ }^{1} \mathrm{H}$ NMR.

${ }^{\mathrm{b}} 0.15 \mathrm{mmol}$ olefin was used.

The aromatic olefins such as chalcones and styrenes gave the best yields (Table 3). No oxidation of aromatic ring of electron rich chalcones has been observed (Table 3, entry 3, 4), although PIDA is a standard reagent for the oxidation of electron rich aromatics. Scale-up of the reaction for trans-4'-methoxychalcone $(0.1 \mathrm{~mol}$ scale) gave product in quantitative yield. 
Table 3. Substrate scope: chalcones and styrenes

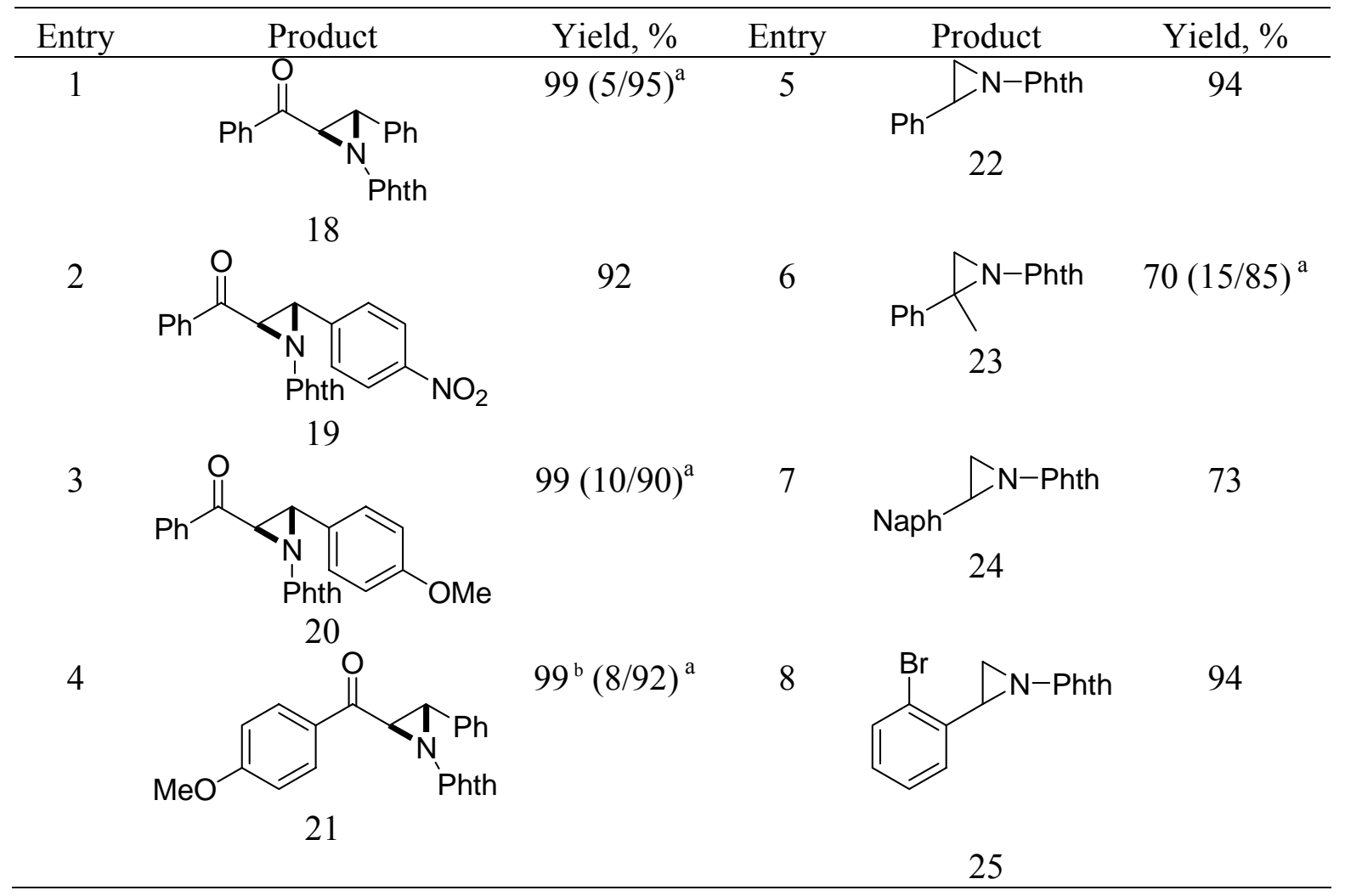

${ }^{\text {a }}$ Ratio of invertomers was determined by ${ }^{1} \mathrm{H}$ NMR.

${ }^{\mathrm{b}} 0.1 \mathrm{~mol}$ scale.

Although dimethyl fumarate gives product in $47 \%$ yield (Table 4, entry 3), dimethyl maleate and maleic anhydride do not react at all. Surprisingly, $N$-methylmaleimide, which is structurally and electronically similar to maleic anhydrate, gives aziridine in $60 \%$ yield (Table 4 , entry 4 ). Haloolefins give the corresponding aziridines only in moderate yields (Table 4, entry 5-8). The reaction with 3,3-dimethylallylbromide was scaled up without significant change in yield (Table 4, entry 5). As well, the aziridination reaction was found to be stereospecific: Z-1,2-dichloro-2butene gives cis-aziridine (Table 4, entry 7), whereas E-olefin gives only the trans-product (Table 4, entry 8). 
Table 4. Substrate scope (continued)

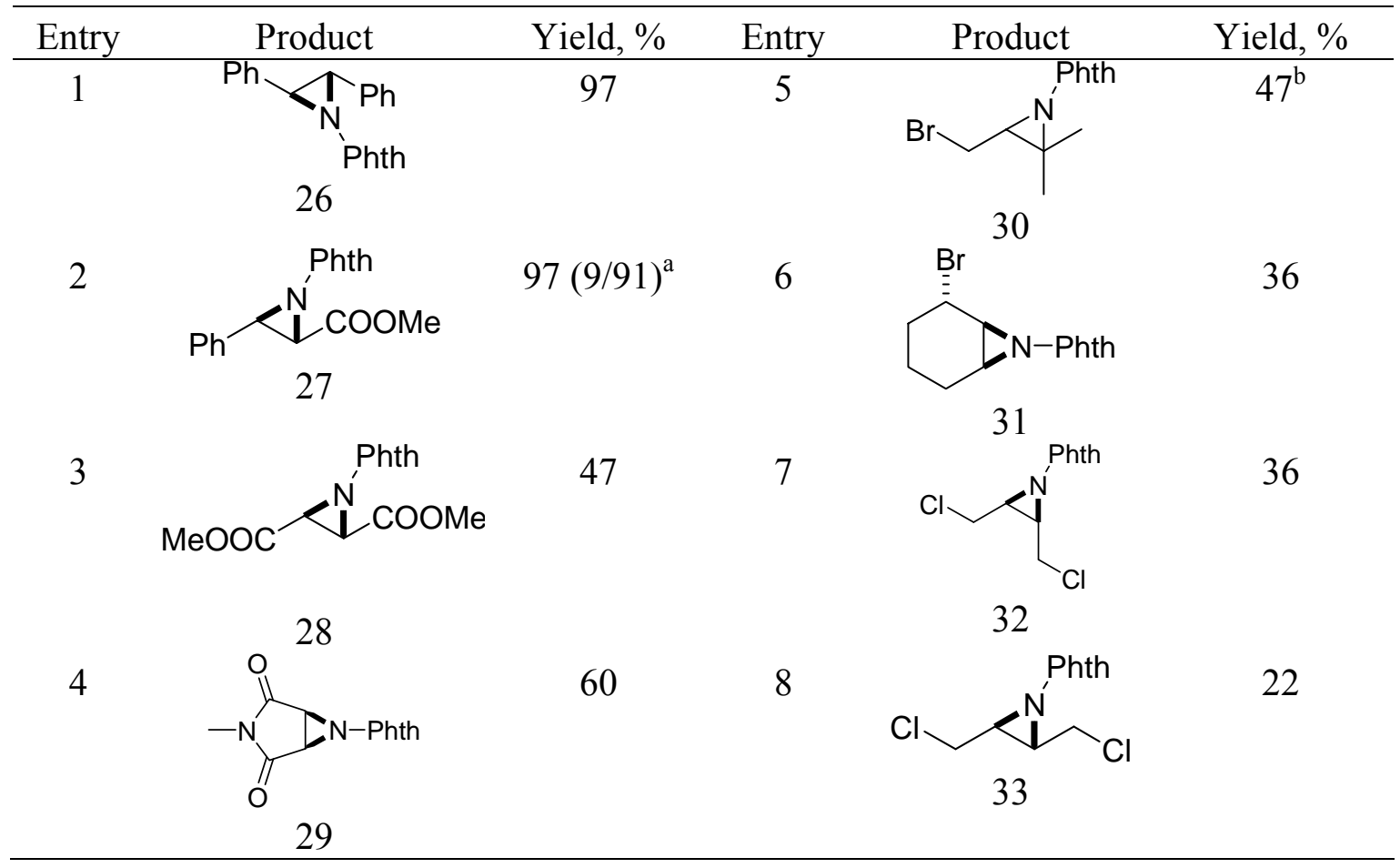

${ }^{\text {a }}$ Ratio of invertomers was determined by ${ }^{1} \mathrm{H}$ NMR.

${ }^{\mathrm{b}} 0.1 \mathrm{~mol}$ scale.

Among the challenging substrates, terminal olefins were found to give lower yields. Excess of olefin increased the yield of 3,3-dimethylbutane aziridine from 7 to $20 \%$ (Table 5, entry 2, 3). The use of fluorinated analog of PIDA (PIFA) did not improve the yield.

Table 5. Terminal olefins in aziridination

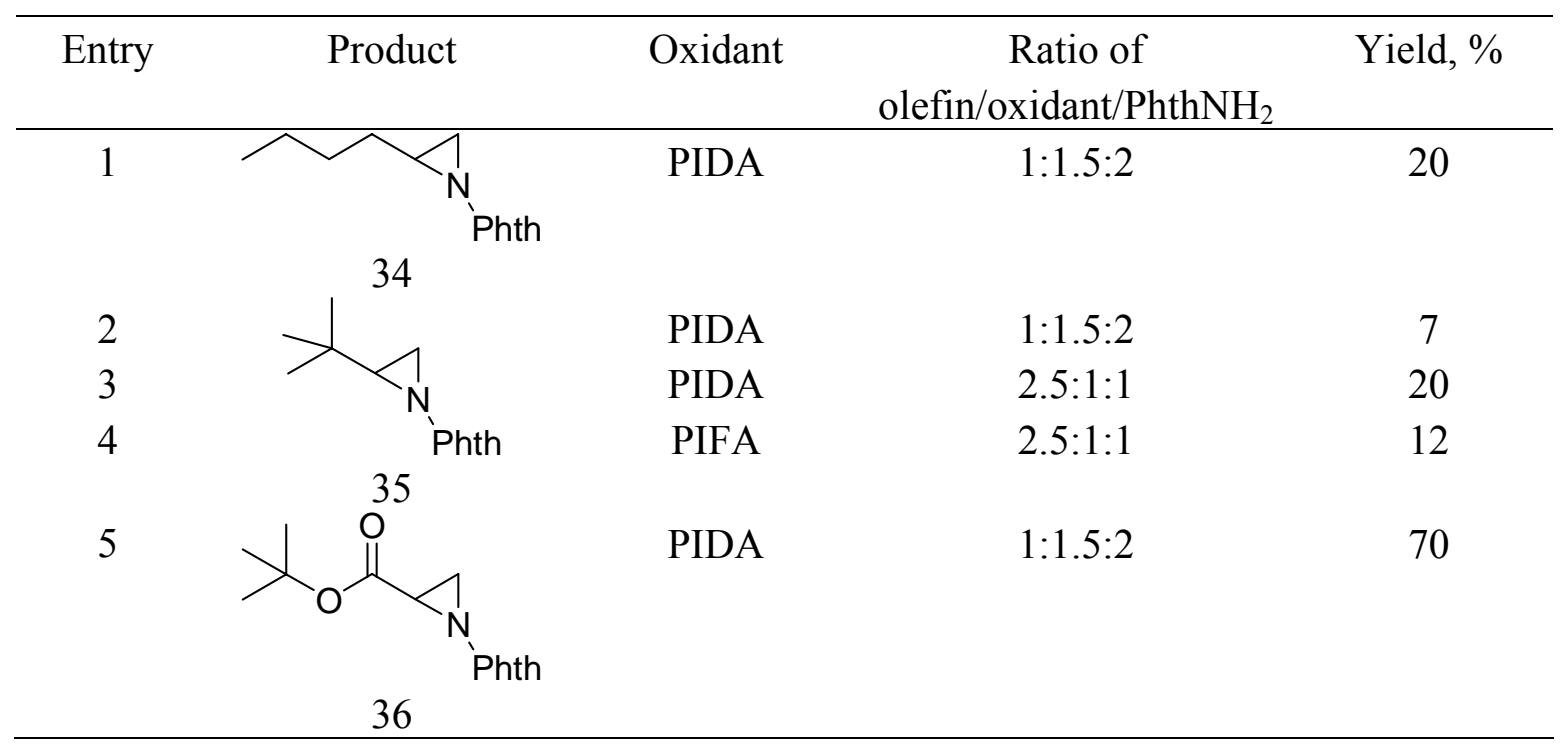


Similar reaction conditions were applied to the imination of sulfoxides. ${ }^{8}$ A number of sulfoxides were converted to the corresponding sulfoximines in moderate to excellent yields (Table 6). In the case of vinylsulfoxide, no reaction at the carbon-carbon double bond has been observed (Table 6, entry 4).

Table 6. Substrate scope in sulfoximination

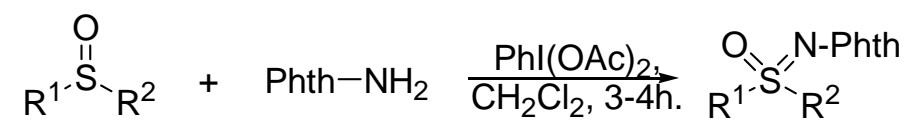

\begin{tabular}{ccc}
\hline Entry & Product & Yield, \% \\
\hline 1 & & 48 \\
& & \\
& & \\
& & \\
& & \\
&
\end{tabular}

The reaction with sulfides was found to be less effective. In the case of benzylphenyl sulfide, the corresponding sulfimine was isolated in small yield together with unreacted starting material. The low yield and efficiency of the reaction can be attributed to the unstable nature of sulfimines, which upon mild heating tend to decompose to the starting sulfides. ${ }^{9}$

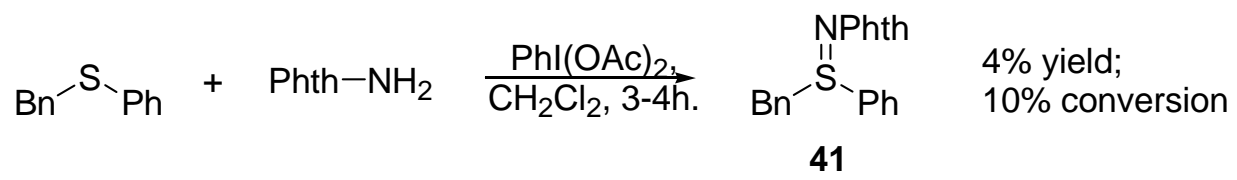

\section{Scheme 3}

In closing, we have found that $\mathrm{PIDA} / \mathrm{PhthNH}_{2}$ combination can be used for aziridination of olefins with a variety of functional groups. The reaction can be scaled up (up to $5 \mathrm{~mol}$ of olefin) without any substantial change in yield. Sulfoxides and sulfides can also be used as nucleophilic traps for the generated nitrenium ions. The non-toxic nature of PIDA together with the simplicity of the developed procedure will facilitate preparation and application of sulfoximines and aziridines, which is the subject of ongoing studies. 


\section{Experimental Section}

General Procedures. All reagents were purchased from Aldrich. ${ }^{1} \mathrm{H}$ and ${ }^{13} \mathrm{C}$ spectra were recorded on either 300 or $400 \mathrm{MHz}$ spectrometer. Column chromatography was carried out using 230-400 mesh silica gel.

\section{General procedure for aziridination or sulfoximination}

(0.5 mmol scale). To the suspension of olefin or sulfoxide $\left(0.5 \mathrm{mmol}, 1\right.$ eq) and $\mathrm{PhthNH}_{2}$ (2eq.) in $2.5 \mathrm{ml}$ dichloromethane, solution of PIDA (1.5eq.) in $2.5 \mathrm{ml}$ of dichloromethane was added dropwise. The reaction mixture was stirred at room temperature for 3-4hrs (monitored by TLC; hexane/ethyl acetate $6 / 4$ or $8 / 2$ (for terminal olefins)). Upon completion, the reaction mixture was quenched with $5 \mathrm{ml}$ of saturated $\mathrm{NaHCO}_{3}$, extracted with ethyl acetate $(3 \times 5 \mathrm{ml})$, dried over sodium sulfate, and concentrated. The products were purified by column chromatography (hexane/ethyl acetate $8 / 2$ ).

(0.1mol scale). To the suspension of olefin $\left(0.1 \mathrm{~mol}\right.$, 1eq) and $\mathrm{PhthNH}_{2}$ (2eq.) in $5 \mathrm{ml}$ of dichloromethane solution of PIDA (1.5eq.) in $20 \mathrm{ml}$ of dichloromethane was added dropwise. The reaction mixture was stirred at room temperature for $4 \mathrm{hrs}$ (monitored by TLC; hexane/ethyl acetate 6/4). The reaction mixture was quenched with $15 \mathrm{ml}$ of saturated $\mathrm{NaHCO}_{3}$, extracted with ethyl acetate $(3 \times 25 \mathrm{ml})$, dried over sodium sulfate, and concentrated. The products were purified by column chromatography (hexane/ethyl acetate $8 / 2$ ).

2-(6-Aza-bicyclo[3.1.0]hex-6-yl)-isoindole-1,3-dione (9). ${ }^{10}{ }^{1} \mathrm{H} \mathrm{NMR}\left(\mathrm{CDCl}_{3}\right) \delta$ : 1.36-1.47 (m, $\left.1 \mathrm{H}, \mathrm{CH}_{2}\right), 1.60-1.67\left(\mathrm{~m}, 1 \mathrm{H}, \mathrm{CH}_{2}\right), 1.75-7.83\left(\mathrm{~m}, 2 \mathrm{H}, \mathrm{CH}_{2}\right), 2.33(\mathrm{dd}, 2 \mathrm{H}, J=8.0,14.0 \mathrm{~Hz}$, $\left.\mathrm{CH}_{2}\right), 3.17$ (s, 2H, CH), 7.65-7.68 (m, 2H, $\left.\mathrm{ArH}\right), 7.74-7.77(2,2 \mathrm{H}, \mathrm{ArH}) .{ }^{13} \mathrm{C} \mathrm{NMR}\left(\mathrm{CDCl}_{3}\right) \delta$ : $19.9,27.7,50.3,123.0,123.7,130.7,134.1,134.4,165.5$.

2-(7-Aza-bicyclo[4.1.0]hept-7-yl)-isoindole-1,3-dione (8). ${ }^{11}{ }^{1} \mathrm{H} \mathrm{NMR}\left(\mathrm{CDCl}_{3}\right) \delta$ : 1.26-1.33 (m, $4 \mathrm{H}, \mathrm{NCHCH}_{2} \mathrm{CH}_{2}$ ), 1.39-1.45 (m, 4H, $\mathrm{NCHCH}_{2} \mathrm{CH}_{2}$ ), 1.94-1.99 (m, 2H, $\mathrm{NCHCH}_{2}$ ), 2.22-2.29 $\left(\mathrm{m}, 2 \mathrm{H}, \mathrm{NCHCH}_{2}\right), 2.73-2.74(\mathrm{~m}, 2 \mathrm{H}, \mathrm{NCH}), 7.65-7.75(\mathrm{~m}, 4 \mathrm{H}, \mathrm{ArH}) .{ }^{13} \mathrm{C} \mathrm{NMR}\left(\mathrm{CDCl}_{3}\right) \delta$ : 20.2, 23.3, 44.0, 122.9, 130.7, 134.0, 165.3.

2-(9-Aza-bicyclo[6.1.0]non-9-yl)-isoindole-1,3-dione (10). ${ }^{11}{ }^{1} \mathrm{H} \mathrm{NMR}\left(\mathrm{CDCl}_{3}\right) \delta$ : $1.20-1.80$ (m, $10 \mathrm{H}, \mathrm{NCHCH}_{2}$ and $\left.\mathrm{NCHCH}_{2}\left(\mathrm{CH}_{2}\right)_{4}\right), 2.51-2.57\left(\mathrm{~m}, 4 \mathrm{H}, \mathrm{NCHCH}\right.$ and $\left.\left.\mathrm{NCHCH}_{2}\right)\right)$, 7.65-7.67 (m, $2 \mathrm{H}, \mathrm{ArH}), 7.73-7.76(\mathrm{~m}, 2 \mathrm{H}, \mathrm{ArH}) .{ }^{13} \mathrm{C} \mathrm{NMR}\left(\mathrm{CDCl}_{3}\right) \delta: 25.6,26.6,26.7,48.2,123.0,130.8$, 134.0, 165.3 .

2-(3-Ethyl-2,2-dimethyl-aziridin-1-yl)-isoindole-1,3-dione (11). ${ }^{11}{ }^{1} \mathrm{H} \mathrm{NMR}\left(\mathrm{CDCl}_{3}\right) \delta: 1.16(\mathrm{t}$, $\left.3 \mathrm{H}, J=7.6 \mathrm{~Hz}, \mathrm{CH}_{3} \mathrm{CH}_{2}\right), 1.29\left(\mathrm{~s}, 3 \mathrm{H},\left(\mathrm{CH}_{3}\right)_{2} \mathrm{C}\right), 1.41\left(\mathrm{~s}, 3 \mathrm{H},\left(\mathrm{CH}_{3}\right)_{2} \mathrm{C}\right), 1.56$ (sept, $1 \mathrm{H}, J=7.2$ $\mathrm{Hz}, \mathrm{CH}_{3} \mathrm{CH}_{2}$ ), 1.86 (sept, 1H, J = 7.2 Hz, $\mathrm{CH}_{3} \mathrm{CH}_{2}$ ), 2.76 (t, 1H, J = 7.2 Hz, NCHEt), 7.66-7.69 $(\mathrm{m}, 2 \mathrm{H}, \mathrm{ArH}), 7.75-7.78(\mathrm{~m}, 2 \mathrm{H}, \mathrm{ArH}) .{ }^{13} \mathrm{C} \mathrm{NMR}\left(\mathrm{CDCl}_{3}\right) \delta: 11.5,19.3,21.2,22.1,47.9,54.4$, $123.0,123.9,134.0,166.4$.

[1-(1,3-Dioxo-1,3-dihydro-isoindol-2-yl)-3-methyl-aziridin-2-yl]-acetonitrile (12, two invertomers in a 17:83 ratio). ${ }^{1} \mathrm{H} \mathrm{NMR}\left(\mathrm{CDCl}_{3}\right) \delta: 1.40\left(\mathrm{~d}, 2.49 \mathrm{H}, \mathrm{J}=6.0 \mathrm{~Hz}, \mathrm{CH}_{3}\right.$, major isomer), $1.52(\mathrm{~d}$, $0.51 \mathrm{H}, J=5.6, \mathrm{CH}_{3}$, minor isomer), 2.60-2.72 (m, 0.34H, $\mathrm{NCHCH}_{3}$, minor isomer), 2.73-2.79 
(m, 1.66H, $\mathrm{NCHCH}_{3}$, major isomer), 2.97-3.05 (m, 1.66H, $(\mathrm{CN}) \mathrm{CH}_{2}$, major isomer), 3.05-3.12 $(\mathrm{m}, 0.34 \mathrm{H},(\mathrm{CN}) \mathrm{CH}$, minor isomer), 7.71-7.75 (m, 1.66H, ArH, major isomer), 7.75-7.77 (m, 0.34H, ArH, minor isomer), 7.78-7.82 (m, 1.66H, $\mathrm{ArH}$, major isomer), 7.86-7.88 (m, 0.34H, ArH, minor isomer). ${ }^{13} \mathrm{C} \mathrm{NMR}\left(\mathrm{CDCl}_{3}\right) \delta: 12.8,17.0,17.02,20.9,42.3,43.0,43.0,43.8,116.3$, $123.4,123.6,123.8,124.5,130.6,130.6,132.7,134.5,134.5,134.6,135.0,166.1,166.2,168.0$. HRMS 241.0847 (Calc. 241.0851 for $\mathrm{C}_{13} \mathrm{H}_{11} \mathrm{~N}_{3} \mathrm{O}_{2}$ ).

2-(2-Hydroxymethyl-3-phenyl-aziridin-1-yl)-isoindole-1,3-dione (13, two invertomers in 36:64 ratio). ${ }^{11} \mathrm{H} \mathrm{NMR}\left(\mathrm{CDCl}_{3}\right) \delta: 7.15-7.45$ (m, 5H, ArH), 7.65-7.80 (m, 4H, ArH), 4.15-4.35 $(\mathrm{m}, 1 \mathrm{H}, \mathrm{PhCH}), 4.02-4.10\left(\mathrm{~m}, 0.36 \mathrm{H}, \mathrm{NCHCH}_{2}\right.$, minor isomer), 3.80-3.90 (m, 0.64H, $\mathrm{NCHCH}_{2}$, major isomer), 3.40-3.60 (m, 1.26H, $\mathrm{CH}_{2} \mathrm{OH}$, major isomer), 3.18-3.22 (m, 0.74H, $\mathrm{CH}_{2} \mathrm{OH}$, minor isomer), 2.57 (t, $0.36 \mathrm{H}, J=6.0 \mathrm{~Hz}, \mathrm{OH}$, minor isomer), 2.69 (bs, 0.64H, OH, major isomer). ${ }^{13} \mathrm{C} \mathrm{NMR}\left(\mathrm{CDCl}_{3}\right) \delta$ : 166.6, 135.8, 134.6, 134.0, 130.5, 129.5, 128.9, 128.7, 128.3, $128.3,127.3,123.5,123.0,62.3,59.3,52.4,49.0,46.7,46.3$.

Acetic acid 1-(1,3-dioxo-1,3-dihydro-isoindol-2-yl)-3-phenyl-aziridin-2-ylmethyl ester (14, two invertomers in a 60:40 ratio). ${ }^{1} \mathrm{H} \mathrm{NMR}\left(\mathrm{CDCl}_{3}\right) \delta: 2.04\left(\mathrm{~s}, 1.2 \mathrm{H}, \mathrm{CH}_{3}\right.$, minor isomer $), 2.19$ (s, $1.8 \mathrm{H}, \mathrm{CH}_{3}$, major isomer), 3.16-3.20 (m, 0.4H, minor isomer), $3.73(\mathrm{~d}, 0.6 \mathrm{H}, J=5.6 \mathrm{~Hz}$, major isomer), $4.14(\mathrm{~d}, 0.4 \mathrm{H}, J=5.6 \mathrm{~Hz}$, minor isomer), $4.24-4.34(\mathrm{~m}, 1.2 \mathrm{H}$, major isomer), 4.39 (dd, $0.4 \mathrm{H}, J=4.4,12.8 \mathrm{~Hz}$, minor isomer), 4.52 (dd, 0.4H, $\mathrm{J}=6.8,12.8 \mathrm{~Hz}$, minor isomer), 4.64 (dd, 0.6H, $J=4.0,11.6 \mathrm{~Hz}$ major isomer), 7.20-7.86 (m, 9H, $\mathrm{ArH}) .{ }^{13} \mathrm{C} \mathrm{NMR}\left(\mathrm{CDCl}_{3}\right) \delta: 20.9$, 21.0, 42.9, 45.6, 47.5, 49.4, 60.6, 64.3, 123.0, 123.3, 123.7, 127.3, 128.3, 128.4, 128.7, 129.0, $129.6,130.1,130.5,130.6,134.1,134.4,134.4,136.0,165.5,166.0,170.7,171.1 . \mathrm{HRMS}$ 336.1106 (Calc. 336.1110 for $\mathrm{C}_{19} \mathrm{H}_{16} \mathrm{~N}_{2} \mathrm{O}_{4}$ ).

1-(1,3-Dioxo-1,3-dihydro-isoindol-2-yl)-2-(hydroxy-phenyl-methyl)-aziridine-2-carboxylic acid methyl ester (15, mixture of two invertomers in 82:18 ratio). ${ }^{11}{ }^{1} \mathrm{H} \mathrm{NMR}\left(\mathrm{CDCl}_{3}\right) \delta$ : 7.60-7.80 (m, 4H, ArH), 7.20-7.45 (m, 5H, ArH), 5.70 (s, 0.82H, PhCHOH, major isomer), 5.70 $\left(\mathrm{s}, 0.18 \mathrm{H}, \mathrm{PhCHOH}\right.$, minor isomer), $3.80(\mathrm{~b}, 1 \mathrm{H}, \mathrm{OH}), 3.64\left(\mathrm{~d}, 0.18 \mathrm{H}, J=2.1 \mathrm{~Hz}, \mathrm{NCH}_{2}\right.$, minor isomer), $3.61\left(\mathrm{~s}, 2.46 \mathrm{H}, \mathrm{CO}_{2} \mathrm{CH}_{3}\right.$, major isomer), $3.58\left(\mathrm{~d}, 0.82 \mathrm{H}, J=1.8 \mathrm{~Hz}, \mathrm{NCH}_{2}\right.$, major isomer), $3.55\left(\mathrm{~s}, 0.54 \mathrm{H}, \mathrm{CO}_{2} \mathrm{CH}_{3}\right.$, major isomer), 2.91 (d, $0.18 \mathrm{H}, J=2.1 \mathrm{~Hz}, \mathrm{NCH}_{2}$, minor isomer), $2.85\left(\mathrm{~d}, 0.82 \mathrm{H}, \mathrm{J}=1.8 \mathrm{~Hz}, \mathrm{NCH}_{2}\right.$, major isomer). ${ }^{13} \mathrm{C} \mathrm{NMR}\left(\mathrm{CDCl}_{3}\right) \delta: 167.0,165.1$, $139.2,134.3,130.2,128.6,128.5,128.4,127.9,127.7,126.8,126.7,123.7,123.4,73.4,70.1$, $53.4,52.1,51.0,40.3$.

2-(Benzenesulfonylamino-phenyl-methyl)-1-(1,3-dioxo-1,3-dihydro-isoindol-2-yl)-aziridine2-carboxylic acid methyl ester (16). ${ }^{11}{ }^{1} \mathrm{H}$ NMR $\left(\mathrm{CDCl}_{3}\right) \delta: 2.12\left(\mathrm{~d}, 1 \mathrm{H}, J=1.6 \mathrm{~Hz}, \mathrm{NCH}_{2}\right)$, $2.35\left(\mathrm{~s}, 3 \mathrm{H}, \mathrm{ArCH}_{3}\right), 3.48\left(\mathrm{~d}, 1 \mathrm{H}, J=1.2 \mathrm{~Hz}, \mathrm{NCH}_{2}\right), 3.50\left(\mathrm{~s}, 3 \mathrm{H}, \mathrm{CO}_{2} \mathrm{CH}_{3}\right), 5.49(\mathrm{~d}, 1 \mathrm{H}, \mathrm{J}=5.6$ $\mathrm{Hz} \mathrm{NH}), 7.13$ (d, 2H, J = 8.0 Hz, ArH), 7.18 (bs, 5H, ArH), 7.38 (d, 1H, J=5.6 Hz, PhCH), 7.63 $(\mathrm{d}, 2 \mathrm{H}, J=8.0 \mathrm{~Hz}, \mathrm{ArH}), 7.69-7.71(\mathrm{~m}, 2 \mathrm{H}, \mathrm{ArH}), 7.77-7.79(\mathrm{~m}, 2 \mathrm{H}, \mathrm{ArH}) .{ }^{13} \mathrm{C} \mathrm{NMR}\left(\mathrm{CDCl}_{3}\right) \delta$ : 21.6, 42.0, 49.1, 53.5, 56.5, 123.6, 127.3, 128.1, 128.3, 128.4, 129.2, 130.1, 134.5, 136.3, 138.9, $142.6,165.5,166.9$.

2-(2-Oxo-7-aza-bicyclo[4.1.0]hept-7-yl)-isoindole-1,3-dione (17). ${ }^{11}{ }^{1} \mathrm{H}$ NMR $\left(\mathrm{CDCl}_{3}\right) \delta$ : 1.72 $1.77\left(\mathrm{~m}, \quad 1 \mathrm{H}, \quad \mathrm{OCCH}_{2}\left(\mathrm{CH}_{2}\right)_{2}\right), \quad 1.94-2.08\left(\mathrm{~m}, 2 \mathrm{H}, \mathrm{OCCH}_{2}\left(\mathrm{CH}_{2}\right)_{2}\right), 2.11-2.18(\mathrm{~m}, 1 \mathrm{H}$, 
$\left.\mathrm{OCCH}_{2}\left(\mathrm{CH}_{2}\right)_{2}\right), 2.53-2.60\left(\mathrm{~m}, 2 \mathrm{H}, \mathrm{O}=\mathrm{CCH}_{2}\right), 3.09$ (d, $\left.1 \mathrm{H}, \mathrm{J}=7.2 \mathrm{~Hz}, \mathrm{NCHCH}_{2}\right), 3.46-3.47(\mathrm{~m}$, $1 \mathrm{H}, \mathrm{O}=\mathrm{CCHN}), 7.60-7.90(\mathrm{~m}, 4 \mathrm{H}, \mathrm{ArH}),{ }^{13} \mathrm{C} \mathrm{NMR}\left(\mathrm{CDCl}_{3}\right) \delta: 18.5,22.3,37.3,46.5,49.3$, $123.5,123.8,130.4,132.9,134.5$.

2-(2-Benzoyl-3-phenyl-aziridin-1-yl)-isoindole-1,3-dione (18, mixture of two invertomers in 5:95 ratio). ${ }^{11}{ }^{1} \mathrm{H} \mathrm{NMR}\left(\mathrm{CDCl}_{3}\right) \delta: 4.26(\mathrm{~d}, 0.05 \mathrm{H}, J=5.2 \mathrm{~Hz}, \mathrm{CH}$, minor isomer), $4.39(\mathrm{~d}$, $0.95 \mathrm{H}, J=4.8 \mathrm{~Hz}, \mathrm{CH}$, major isomer), 4.89 (d, 0.95H, $J=4.8 \mathrm{~Hz}, \mathrm{CH}$, major isomer), 5.54 (d, $0.05 \mathrm{H}, J=5.2 \mathrm{~Hz}, \mathrm{CH}$, minor isomer), 7.35-8.32 (m, $10 \mathrm{H}, \mathrm{ArH}) .{ }^{13} \mathrm{C} \mathrm{NMR}\left(\mathrm{CDCl}_{3}\right) \delta$ (major isomer): $50.8,48.8,123.3,127.4,128.8,128.8,128.9,128.9,130.4,133.8,134.1,135.3,137.5$, $164.8,190.7$.

2-[2-Benzoyl-3-(4-nitro-phenyl)-aziridin-1-yl]-isoindole-1,3-dione (19). ${ }^{1} \mathrm{H} \mathrm{NMR}\left(\mathrm{CDCl}_{3}\right) \delta$ : $4.38(\mathrm{~d}, 1 \mathrm{H}, J=4.8 \mathrm{~Hz}, \mathrm{PhCH}), 4.75(\mathrm{~d}, 1 \mathrm{H}, J=4.8 \mathrm{~Hz}, \mathrm{PhC}(=\mathrm{O}) \mathrm{CH}), 7.52-7.75$ (m, 10H, $\operatorname{ArH})$, 8.09-8.11 (m, 2H, ArH), 8.25-8.28 (m, 2H, ArH),. ${ }^{13} \mathrm{C} \mathrm{NMR}\left(\mathrm{CDCl}_{3}\right)$ 8: 49.1, 49.5, 123.1, 127.2, $128.6,128.7,128.7,128.7,130.2,133.6,134.0,135.2,137.3,164.7,189.8$. HRMS 413.1003 (Calc. 413.1012 for $\mathrm{C}_{23} \mathrm{H}_{15} \mathrm{~N}_{3} \mathrm{O}_{5}$ ).

2-[2-Benzoyl-3-(4-methoxy-phenyl)-aziridin-1-yl]-isoindole-1,3-dione (20, mixture of two invertomers in 10:90 ratio). ${ }^{1} \mathrm{H} \mathrm{NMR}\left(\mathrm{CDCl}_{3}\right) \delta: 3.70\left(\mathrm{~s}, 0.3 \mathrm{H}, \mathrm{CH}_{3}\right.$, minor isomer), $3.80(\mathrm{~s}$, 2.7H, $\mathrm{CH}_{3}$, major isomer), $4.20(\mathrm{~d}, 0.1 \mathrm{H}, \mathrm{J}=5.6 \mathrm{~Hz}, \mathrm{CH}$, minor isomer), 4.39 (d, $0.9 \mathrm{H}, J=4.8$ $\mathrm{Hz}, \mathrm{CH}$, major isomer), $4.63(\mathrm{~d}, 0.9 \mathrm{H}, \mathrm{J}=4.8 \mathrm{~Hz}, \mathrm{CH}$, major isomer), $5.43(\mathrm{~d}, 0.1 \mathrm{H}, \mathrm{J}=5.6 \mathrm{~Hz}$, $\mathrm{CH}$, minor isomer), 6.77-8.31 (m, $13 \mathrm{H}, \mathrm{ArH}) .{ }^{13} \mathrm{C} \mathrm{NMR}\left(\mathrm{CDCl}_{3}\right) \delta: 44.6,48.7,50.6,51.2,55.3$, $55.5,114.0,114.3,121.6,123.2,123.6,124.6,127.3,128.6,128.9,128.9$, 129.0, 130.4, 130.9, $132.9,133.7,134.1,134.2,134.3,135.2,137.5,160.0,160.3,164.8,168.2,190.8,193.3 . \mathrm{HRMS}$ 398.1264 (Calc. 398.1267 for $\mathrm{C}_{24} \mathrm{H}_{18} \mathrm{~N}_{2} \mathrm{O}_{4}$ ).

2-[2-(4-Methoxy-benzoyl)-3-phenyl-aziridin-1-yl]-isoindole-1,3-dione (21, mixture of two invertomers in 8:92 ratio)). ${ }^{1} \mathrm{H} \mathrm{NMR}\left(\mathrm{CDCl}_{3}\right) \delta: 3.89\left(\mathrm{~s}, 2.76 \mathrm{H}, \mathrm{CH}_{3}\right.$, major isomer), 3.91 (s, $0.24 \mathrm{H}, \mathrm{CH}_{3}$, minor isomer), $4.27(\mathrm{~d}, 0.08 \mathrm{H}, J=5.2 \mathrm{~Hz}, \mathrm{CH}$, minor isomer), $4.36(\mathrm{~d}, 0.92 \mathrm{H}, J=$ $4.8 \mathrm{~Hz}, \mathrm{CH}$, major isomer), $4.69(\mathrm{~d}, 0.9 \mathrm{H}, J=4.8 \mathrm{~Hz}, \mathrm{CH}$, major isomer), $5.50(\mathrm{~d}, 0.08 \mathrm{H}, J=5.2$ $\mathrm{Hz}, \mathrm{CH}$, minor isomer), 6.97-8.33 (m, $13 \mathrm{H}, \mathrm{ArH}) .{ }^{13} \mathrm{C} \mathrm{NMR}\left(\mathrm{CDCl}_{3}\right) \delta$ (major isomer): 48.7 , 50.3, 55.8, 123.3, 123.8, 127.4, 128.7, 128.9, 130.5, 131.3, 134.1, 134.5, 136.0, 164.3, 164.8, 188.8. HRMS 398.1264 (Calc. 398.1267 for $\mathrm{C}_{24} \mathrm{H}_{18} \mathrm{~N}_{2} \mathrm{O}_{4}$ ).

2-(2-Phenyl-aziridin-1-yl)-isoindole-1,3-dione (22). ${ }^{12}{ }^{1} \mathrm{H} \mathrm{NMR}\left(\mathrm{CDCl}_{3}\right) \delta: 2.80(\mathrm{dd}, 1 \mathrm{H}, J=$ 2.4, $6.0 \mathrm{~Hz}, \mathrm{CH}_{2}$ ), 2.90 (dd, $1 \mathrm{H}, J=2.4,8.0 \mathrm{~Hz}, \mathrm{CH}_{2}$ ), 3.61 (dd, $\left.1 \mathrm{H}, J=6.0,8.0 \mathrm{~Hz}, \mathrm{CH}\right), 7.30$ $7.47(\mathrm{~m}, 5 \mathrm{H}, \mathrm{ArH}), 7.68-7.80(\mathrm{~m}, 4 \mathrm{H}, \mathrm{ArH}) .{ }^{13} \mathrm{C} \mathrm{NMR}\left(\mathrm{CDCl}_{3}\right)$ 8: 39.9, 44.7, 123.3, 123.8, $127.4,128.2,128.7,130.5,134.3,134.4,136.6$.

2-(2-Methyl-2-phenyl-aziridin-1-yl)-isoindole-1,3-dione (23, two invertomers in 85:15 ratio). ${ }^{12}{ }^{1} \mathrm{H} \mathrm{NMR}\left(\mathrm{CDCl}_{3}\right) \delta: 1.64\left(\mathrm{~s}, 2.55 \mathrm{H}, \mathrm{CH}_{3}\right.$, major isomer), $1.82\left(\mathrm{~s}, 0.45 \mathrm{H}, \mathrm{CH}_{3}\right.$, minor isomer), $2.68\left(\mathrm{~d}, 0.15 \mathrm{H}, J=3.6 \mathrm{~Hz}, \mathrm{CH}_{2}\right.$, minor isomer), $2.87\left(\mathrm{~d}, 0.85 \mathrm{H}, J=2.8 \mathrm{~Hz}, \mathrm{CH}_{2}\right.$, major isomer), $3.13\left(\mathrm{~d}, 1 \mathrm{H}, J=2.8 \mathrm{~Hz}, \mathrm{CH}_{2}\right.$, major isomer), $4.26\left(\mathrm{~d}, 1 \mathrm{H}, J=3.6 \mathrm{~Hz}, \mathrm{CH}_{2}\right.$, minor isomer), 7.15-7.83 (m, 9H, ArH). ${ }^{13} \mathrm{C} \mathrm{NMR}\left(\mathrm{CDCl}_{3}\right) \delta: 122.8,123.2,127.6,127.7,128.2,128.5$, $128.6,129.3,130.8,133.9,134.2,141.8,166.0,166.4$. 
2-(2-Naphthalen-2-yl-aziridin-1-yl)-isoindole-1,3-dione (24). ${ }^{5}{ }^{1} \mathrm{H} \mathrm{NMR}\left(\mathrm{CDCl}_{3}\right) \delta$ : 2.95 (dd, $\left.1 \mathrm{H}, J=2.4,6.0 \mathrm{~Hz}, \mathrm{CH}_{2}\right), 3.02\left(\mathrm{dd}, 1 \mathrm{H}, \mathrm{J}=2.4,8.0 \mathrm{~Hz}, \mathrm{CH}_{2}\right), 3.82(\mathrm{dd}, 1 \mathrm{H}, J=5.6,8.0 \mathrm{CH})$, 7.47-7.49 (m, 2H, ArH), 7.58-7.60 (m, 1H, ArH), 7.70-7.88 (m, 8H, ArH). ${ }^{13} \mathrm{C} \mathrm{NMR}\left(\mathrm{CDCl}_{3}\right) \delta$ : 40.2 , 44.9, 123.4, 123.8, 125.0, 126.3, 126.5, 126.7, 127.9, 128.1, 128.6, 130.6, 134.4, 134.6, 165.3 .

2-[2-(2-Bromo-phenyl)-aziridin-1-yl]-isoindole-1,3-dione (25). ${ }^{1} \mathrm{H}$ NMR $\left(\mathrm{CDCl}_{3}\right) \delta$ : 2.54 (dd, $\left.1 \mathrm{H}, J=2.0,6.0 \mathrm{~Hz}, \mathrm{CH}_{2}\right), 3.05\left(\mathrm{dd}, 1 \mathrm{H}, J=2.0,8.0 \mathrm{~Hz}, \mathrm{CH}_{2}\right), 3.79(\mathrm{dd}, 1 \mathrm{H}, J=6.0,8.0 \mathrm{~Hz}$, $\mathrm{CH}), 7.17$ (dt, $1 \mathrm{H}, J=1.2,7.6 \mathrm{~Hz}, \mathrm{ArH}), 7.33$ (t, 1H, $J=7.6 \mathrm{~Hz}, \mathrm{ArH}), 7.54-7.60$ (m, 2H, ArH). ${ }^{13} \mathrm{C} \mathrm{NMR}\left(\mathrm{CDCl}_{3}\right) \delta: 40.6,44.8,123.3,123.5,127.8,128.7,129.3,130.5,132.3,134.3,136.6$, 165.1. HRMS 341.995 (Calc. 342.0004 for $\mathrm{C}_{16} \mathrm{H}_{11} \mathrm{BrN}_{2} \mathrm{O}_{2}$ ).

2-(2,3-Diphenyl-aziridin-1-yl)-isoindole-1,3-dione (26). ${ }^{13}{ }^{1} \mathrm{H} \mathrm{NMR}\left(\mathrm{CDCl}_{3}\right) \delta: 3.97$ (d, $1 \mathrm{H}, J=$ $6.0 \mathrm{~Hz}, \mathrm{PhC}(=\mathrm{O}) \mathrm{CH}), 4.95(\mathrm{~d}, 1 \mathrm{H}, J=5.6 \mathrm{~Hz}, \mathrm{PhCH}), 7.24-7.65$ (m, 14H. ArH). ${ }^{13} \mathrm{C}$ NMR $\left(\mathrm{CDCl}_{3}\right) \delta: 46.7,53.8,123.1,127.4,128.3,128.4,128.8,128.9,129.5,130.3,131.4,134.1$, $136.7,165.7$.

1-(1,3-Dioxo-1,3-dihydro-isoindol-2-yl)-3-phenyl-aziridine-2-carboxylic acid methyl ester (27, mixture of two invertomers in 91:9 ratio). ${ }^{11}{ }^{1} \mathrm{H} \mathrm{NMR}\left(\mathrm{CDCl}_{3}\right) \delta: 3.53(\mathrm{~d}, 0.91 \mathrm{H}, \mathrm{J}=8.4 \mathrm{~Hz}$, $\mathrm{CH}$, major isomer), $3.75\left(\mathrm{~s}, 2.73 \mathrm{H}, \mathrm{CH}_{3}\right.$, major isomer), $3.89\left(\mathrm{~s}, 0.27 \mathrm{H}, \mathrm{CH}_{3}\right.$, minor isomer), 4.08 (d, $0.09 \mathrm{H}, J=5.6 \mathrm{~Hz}, \mathrm{CH}$, minor isomer), $4.39(\mathrm{~d}, 0.91 \mathrm{H}, J=8.4 \mathrm{~Hz}, \mathrm{CH}$, major isomer), 4.62 (d, 0.09H, $J=5.6 \mathrm{~Hz}, \mathrm{CH}$, minor isomer), 7.24-7.87 (m, 9H, $\mathrm{ArH}) .{ }^{13} \mathrm{C} \mathrm{NMR}\left(\mathrm{CDCl}_{3}\right) \delta$ : (major isomer) $46.2,49.8,53.1,123.3,127.4,128.8,130.4,134.3,134.6,164.8$ and (minor isomer) $31.1,42.4,50.2,123.3,123.7,128.5,129.6,134.3,134.4,166.9$.

trans-1-(1,3-Dioxo-1,3-dihydro-isoindol-2-yl)-aziridine-2,3-dicarboxylic acid dimethyl ester (28). ${ }^{11} \mathrm{H} \mathrm{NMR}_{\left(\mathrm{CDCl}_{3}\right)}$ ): $3.63\left(\mathrm{~d}, 1 \mathrm{H}, \mathrm{J}=4.5 \mathrm{~Hz}, \mathrm{MeO}_{2} \mathrm{CCH}\right), 3.76$ (s, 3H, $\left.\mathrm{CH}_{3} \mathrm{CO}_{2}\right), 3.88$ (s, $\left.3 \mathrm{H}, \mathrm{CH}_{3} \mathrm{CO}_{2}\right), 3.98\left(\mathrm{~d}, 1 \mathrm{H}, J=4.5 \mathrm{~Hz}, \mathrm{MeO}_{2} \mathrm{CCH}\right), 7.69-7.80(\mathrm{~m}, 4 \mathrm{H}, \mathrm{ArH}) .{ }^{13} \mathrm{C} \mathrm{NMR}\left(\mathrm{CDCl}_{3}\right)$ $\delta: 42.9,44.9,53.4,53.5,123.6,130.1,134.5,164.3,165.5,166.7$.

2-(3-Methyl-2,4-dioxo-3,6-diaza-bicyclo[3.1.0]hex-6-yl)-isoindole-1,3-dione (29). ${ }^{1} \mathrm{H}$ NMR $\left(\mathrm{CDCl}_{3}\right) \delta: 2.97\left(\mathrm{~s}, 3 \mathrm{H}, \mathrm{CH}_{3}\right), 4.33(\mathrm{~d}, 2 \mathrm{H}, \mathrm{J}=0.4 \mathrm{~Hz}, \mathrm{CH}), 7.76-7.89$ (m, 4H, ArH). ${ }^{13} \mathrm{C}$ NMR $\left(\mathrm{CDCl}_{3}\right) \delta: 25.1,42.3,123.8,124.0,129.7,132.9,134.5,135.1,164.2,164.7,168.0,169.3$. HRMS 271.0596 (Calc. 271.0593 for $\mathrm{C}_{13} \mathrm{H}_{9} \mathrm{~N}_{3} \mathrm{O}_{4}$ ).

2-(3-Bromomethyl-2,2-dimethyl-aziridin-1-yl)-isoindole-1,3-dione (30, two invertomers in a 17:83 ratio). ${ }^{1} \mathrm{H}$ NMR $\left(\mathrm{CDCl}_{3}\right) \delta: 1.36\left(\mathrm{~s}, 2.49 \mathrm{H}, \mathrm{CH}_{3}\right.$, major isomer), $1.48\left(\mathrm{~s}, 2.49 \mathrm{H}, \mathrm{CH}_{3}\right.$, major isomer), $1.95\left(\mathrm{~s}, 0.51 \mathrm{H}, \mathrm{CH}_{3}\right.$, minor isomer), $2.04\left(\mathrm{~s}, 0.51 \mathrm{H}, \mathrm{CH}_{3}\right.$, minor isomer), 3.18 (dd, $0.83 \mathrm{H}, J=1.6,10.0 \mathrm{~Hz}, \mathrm{CH}_{2}$, major isomer), 3.19 (dd, 0.83H, $J=10.0,23.6 \mathrm{~Hz}, \mathrm{CH}_{2}$, major isomer), 3.58 (dd, $0.17 \mathrm{H}, J=5.6,11.2 \mathrm{~Hz}, \mathrm{CH}_{2}$, minor isomer), 3.69 (dd, $0.17 \mathrm{H}, J=3.6,6.0 \mathrm{~Hz}$, $\mathrm{CH}$, minor isomer), $3.80\left(\mathrm{dd}, 0.17 \mathrm{H}, J=3.2,11.2 \mathrm{~Hz}, \mathrm{CH}_{2}\right.$, minor isomer), 4.02 (dd, $0.83 \mathrm{H}, J=$

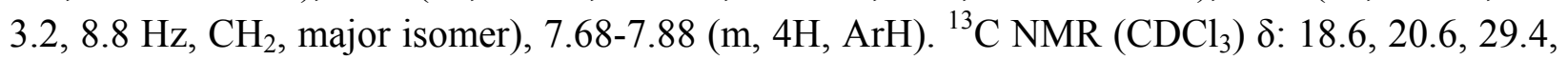
31.4, 31.8, 32.3, 49.7, 52.1, 67.8, 70.0, 123.3, 123.8, 130.4, 130.8, 134.3, 134.6, 165.9, 166.8. HRMS 308.0162 (Calc. 308.0160 for $\mathrm{C}_{13} \mathrm{H}_{13} \mathrm{BrN}_{2} \mathrm{O}_{2}$ ).

2-(anti-2-Bromo-7-azabicyclo[4.1.0]hept-7-yl)-isoindole-1,3-dione (31). ${ }^{1} \mathrm{H} \mathrm{NMR}\left(\mathrm{CDCl}_{3}\right) \delta$ : 1.39-1.44 (m, 1H, $\left.\mathrm{CH}_{2}\right), 1.66-1.82\left(\mathrm{~m}, 2 \mathrm{H}, \mathrm{CH}_{2}\right), 1.96-2.04\left(\mathrm{~m}, 1 \mathrm{H}, \mathrm{CH}_{2}\right), 2.11-2.29(\mathrm{~m}, 2 \mathrm{H}$, 
$\left.\mathrm{CH}_{2}\right), 2.93\left(\mathrm{t}, 1 \mathrm{H}, J=6.3 \mathrm{~Hz}, \mathrm{CH}_{2} \mathrm{CHN}\right), 3.15(\mathrm{~d}, 1 \mathrm{H}, J=7.2 \mathrm{~Hz}, \mathrm{BrCHCHN}), 4.90(\mathrm{~m}, 1 \mathrm{H}$, $\mathrm{BrCH}), 7.67-7.76(\mathrm{~m}, 4 \mathrm{H}, \mathrm{ArH}) .{ }^{13} \mathrm{C} \mathrm{NMR}\left(\mathrm{CDCl}_{3}\right) \delta: 16.3,21.9,29.6,44.4,47.0,48.7,123.3$, 125.9, 130.6, 134.3, 136.2, 165.0. HRMS 320.0168 (Calc. 320.0160 for $\mathrm{C}_{14} \mathrm{H}_{13} \mathrm{BrN}_{2} \mathrm{O}_{2}$ ). cis-2-(2,3-Bis-chloromethylaziridin-1-yl)-isoindole-1,3-dione (32). ${ }^{11}{ }^{1} \mathrm{H}$ NMR $\left(\mathrm{CDCl}_{3}\right) \delta$ : 3.20-3.26 (m, 2H, $\left.\mathrm{ClCH}_{2}\right), 3.51-3.58\left(\mathrm{~m}, 2 \mathrm{H}, \mathrm{ClCH}_{2}\right), 4.07-4.11$ (m, 2H, $\left.\mathrm{NCHCH}\right), 7.60-7.80$ (m, 4H, ArH). ${ }^{13} \mathrm{C} \mathrm{NMR}\left(\mathrm{CDCl}_{3}\right) \delta: 40.2,48.1,123.6,130.3,134.6,164.8$.

trans-2-(2,3-Bis-chloromethylaziridin-1-yl)-isoindole-1,3-dione (33). ${ }^{11} \mathrm{H} \mathrm{NMR}\left(\mathrm{CDCl}_{3}\right) \delta$ : $2.98\left(\mathrm{dt}, 1 \mathrm{H}, J=5.1,7.5 \mathrm{~Hz}, \mathrm{ClCH}_{2}\right), 3.48-3.59$ (m, 3H, $\left.\mathrm{ClCH}_{2}\right), 4.00-4.06(\mathrm{~m}, 2 \mathrm{H}, \mathrm{NCHCH})$, 7.71-7.89 (m, 4H, ArH). ${ }^{13} \mathrm{C} \mathrm{NMR}\left(\mathrm{CDCl}_{3}\right) \delta: 40.7,43.5,46.2,47.0,123.6,130.5,134.5,165.9$. 2-(2-Butyl-aziridin-1-yl)-isoindole-1,3-dione (34). ${ }^{1} \mathrm{H} \mathrm{NMR}\left(\mathrm{CDCl}_{3}\right) \delta: 0.96(\mathrm{t}, 3 \mathrm{H}, J=7.2 \mathrm{~Hz}$, $\left.\mathrm{CH}_{3}\right), 1.38-1.46\left(\mathrm{~m}, 2 \mathrm{H}, \mathrm{CH}_{2}\right), 1.53-1.77\left(\mathrm{~m}, 4 \mathrm{H}, \mathrm{CH}_{2}\right), 2.28\left(\mathrm{dd}, 1 \mathrm{H}, J=2.0,5.6 \mathrm{~Hz}, \mathrm{NCH}_{2}\right)$, 2.45 (dd, 1H, $J=2.0,7.6 \mathrm{~Hz}, \mathrm{CH}_{2}$ ), 2.61 (quint., $\left.1 \mathrm{H}, J=6.8 \mathrm{~Hz}, \mathrm{NCH}\right), 7.66-7.70$ (m, 2H, ArH), 7.73-7.78 (m, 2H, ArH). ${ }^{13} \mathrm{C} \mathrm{NMR}\left(\mathrm{CDCl}_{3}\right) \delta: 14.2,22.8,28.7,31.9,38.4,43.5,123.1,130.6$, 134.2, 165.4. HRMS 244.1215 (Calc. 244.1212 for $\mathrm{C}_{14} \mathrm{H}_{16} \mathrm{~N}_{2} \mathrm{O}_{2}$ ).

2-(2-tert-Butyl-aziridin-1-yl)-isoindole-1,3-dione (35). ${ }^{1} \mathrm{H} \mathrm{NMR}\left(\mathrm{CDCl}_{3}\right) \delta: 1.06\left(\mathrm{~s}, 9 \mathrm{H}, \mathrm{CH}_{3}\right)$, $2.31\left(\mathrm{dd}, J=2.4,8.0 \mathrm{~Hz}, \mathrm{CH}_{2}\right), 2.45\left(\mathrm{dd}, J=2.4,6.0 \mathrm{~Hz}, \mathrm{CH}_{2}\right), 2.53(\mathrm{dd}, J=6.0,8.0 \mathrm{~Hz}, \mathrm{CH})$, 7.66-7.68 (m, 2H, ArH), 7.74-7.76 (m, 2H, ArH). ${ }^{13} \mathrm{C} \mathrm{NMR}\left(\mathrm{CDCl}_{3}\right) \delta: 26.9,30.9,35.3$, 51.6, 123.1, 130.6, 134.2, 165.5. HRMS 244.1207 (Calc. 244.1212 for $\mathrm{C}_{14} \mathrm{H}_{16} \mathrm{~N}_{2} \mathrm{O}_{2}$ ).

1-(1,3-Dioxo-1,3-dihydro-isoindol-2-yl)-aziridine-2-carboxylic acid tert-butyl ester (36). ${ }^{14}{ }^{1} \mathrm{H}$ NMR $\left(\mathrm{CDCl}_{3}\right) \delta: 1.39\left(\mathrm{~s}, 1.8 \mathrm{H}, \mathrm{C}\left(\mathrm{CH}_{3}\right)_{3}\right)$, minor isomer), $1.54\left(\mathrm{~s}, 7.2 \mathrm{H}, \mathrm{C}\left(\mathrm{CH}_{3}\right)_{3}\right)$, major isomer), $2.77(\mathrm{~d}, 0.8 \mathrm{H}, J=7.6 \mathrm{~Hz}, \mathrm{CH}$, major isomer), $2.81(\mathrm{~d}, 0.8 \mathrm{H}, J=5.6 \mathrm{~Hz}, \mathrm{CH}), 3.16(\mathrm{t}$, $0.2 \mathrm{H}, J=6.0 \mathrm{~Hz}, \mathrm{CH}$, minor isomer), $3.20(\mathrm{~d}, 0.2 \mathrm{H}, J=6.4 \mathrm{~Hz}, \mathrm{CH}$, minor isomer), 3.08 (dd, $0.8 \mathrm{H}, J=6.0,7.6 \mathrm{~Hz}, \mathrm{CH}$, minor isomer), 7.65-7.80 (m, $2 \mathrm{H}, \mathrm{ArH}) .{ }^{13} \mathrm{C} \mathrm{NMR}\left(\mathrm{CDCl}_{3}\right) \delta: 27.8$, 28.2, 36.2, 36.6, 39.1, 41.1, 82.7, 82.7, 123.1, 123.5, 130.2, 130.4, 134.1, 134.4, 164.7, 164.9, 165.9, 167.3.

$N$-(1,3-Dioxo-1,3-dihydro-isoindol-2-yl)-dimethylsulfoximine (37). ${ }^{11}{ }^{1} \mathrm{H}$ NMR $\left(\mathrm{CDCl}_{3}\right) \delta$ : $3.29\left(\mathrm{~s}, 6 \mathrm{H}, \mathrm{CH}_{3} \mathrm{SCH}_{3}\right), 7.71-7.75(\mathrm{~m}, 2 \mathrm{H}, \mathrm{ArH}), 7.82-7.86(\mathrm{~m}, 2 \mathrm{H}, \mathrm{ArH}) .{ }^{13} \mathrm{C} \mathrm{NMR}\left(\mathrm{CDCl}_{3}\right) \delta$ : $41.4,123.5,130.9,134.3,167.6$.

$\boldsymbol{N}$-(1,3-Dioxo-1,3-dihydro-isoindol-2-yl)-tetramethylene sulfoximine (38). ${ }^{11}{ }^{1} \mathrm{H} \mathrm{NMR}\left(\mathrm{CDCl}_{3}\right)$ $\delta: 2.30-2.50\left(\mathrm{~m}, 4 \mathrm{H}, \mathrm{SCH}_{2}\left(\mathrm{CH}_{2}\right)_{2}\right), 3.17-3.26\left(\mathrm{~m}, 2 \mathrm{H}, \mathrm{SCH}_{2}\right), 3.67-3.76\left(\mathrm{~m}, 2 \mathrm{H}, \mathrm{SCH}_{2}\right), 7.68-$ $7.74(\mathrm{~m}, 2 \mathrm{H}, \mathrm{ArH}), 7.80-7.85$ (m, 2H, ArH). ${ }^{13} \mathrm{C} \mathrm{NMR}\left(\mathrm{CDCl}_{3}\right)$ 8: 24.1, 52.9, 123.4, 130.9, 134.2, 167.6 .

N-(1,3-Dioxo-1,3-dihydro-isoindol-2-yl)-methyl-(p-tolyl)sulfoximine (39). ${ }^{11}{ }^{1} \mathrm{H} \mathrm{NMR}\left(\mathrm{CDCl}_{3}\right)$ $\delta: 2.44\left(\mathrm{~s}, 3 \mathrm{H}, \mathrm{ArCH}_{3}\right), 3.34\left(\mathrm{~s}, 3 \mathrm{H}, \mathrm{SCH}_{3}\right), 7.38(\mathrm{~d}, 2 \mathrm{H}, \mathrm{J}=8.4 \mathrm{~Hz}, \mathrm{ArH}), 7.64-7.69$ (m, 2H, $\operatorname{ArH}), 7.74-7.78(\mathrm{~m}, 2 \mathrm{H}, \mathrm{ArH}), 8.11(\mathrm{~d}, 2 \mathrm{H}, J=8.4 \mathrm{~Hz}, \mathrm{ArH}) .{ }^{13} \mathrm{C} \mathrm{NMR}\left(\mathrm{CDCl}_{3}\right) \delta: 21.9,42.8$, $123.3,129.7,130.2,130.9,133.4,134.0,145.6,167.2$.

$\mathbf{N}$-(1,3-Dioxo-1,3-dihydro-isoindol-2-yl)-phenylvinylsulfoximine (40). ${ }^{11}{ }^{1} \mathrm{H} \mathrm{NMR}\left(\mathrm{CDCl}_{3}\right) \delta$ : $6.22\left(\mathrm{dd}, 1 \mathrm{H}, J=0.9,9.6 \mathrm{~Hz}, \mathrm{C}=\mathrm{CH}_{2}\right), 6.53\left(\mathrm{dd}, 1 \mathrm{H}, J=0.9,16.5 \mathrm{~Hz}, \mathrm{C}=\mathrm{CH}_{2}\right), 6.86(\mathrm{dd}, 1 \mathrm{H}, J=$ 9.6, $\left.16.5 \mathrm{~Hz}, \mathrm{HC}=\mathrm{CH}_{2}\right), 7.55-7.82(\mathrm{~m}, 7 \mathrm{H}, \mathrm{ArH}), 8.20-8.23(\mathrm{~m}, 2 \mathrm{H}, \mathrm{ArH}) .{ }^{13} \mathrm{C} \mathrm{NMR}\left(\mathrm{CDCl}_{3}\right) \delta$ : $123.3,129.6,129.6,130.8,132.2,134.1,134.5,135.2,135.9,167.1$. 


\section{Acknowledgements}

We thank NSERC, CFI, ORDCF, ACS-PRF, the University of Toronto, Affinium Pharmaceuticals, and Amgen for financial support. AY is a Cottrell Scholar of Research Corporation.

\section{References and Footnotes}

1. (a) Modern Amination Methods; Ricci, A., Ed.; Wiley-VCH: Weinheim, 2000. (b) Johannsen, M.; Jørgensen, K.A. Chem. Rev. 1998, 98, 1689.

2. (a) Siu, T.; Yudin, A.K. J. Am. Chem. Soc. 2002, 124, 530. (b) Siu, T.; Yudin, A. K. Org. Lett. 2002, 4(11), 1839.

3. (a) Anderson, D.J.; Gilchrist, T.L.; Horwell, D.C.; Rees, C.W. J. Chem. Soc. C 1970, 4, 576.

(b) Felix, D.; Müller, R.K.; Horn, W.; Joos, R.; Schreiber, J.; Eschenmoser, A. Helv. Chim. Acta 1972, 55, 1276.

4. Lead (IV) acetate: Mihailović, M.L.; Čeković, Ž. In Encyclopedia of Reagents for Organic Synthesis; Paquett, L.A., Ed.; Wiley: New York, 1995; Vol. 5, p 2554.

5. During preparation of this manuscript similar aziridination protocol has been reported: Li, J.; Liang, J.-L.; Chan, P.W.H.; Che, C.-M. Tetrahedron Lett. 2004, 45, 2685.

6. (a) Atkinson, R.S.; Grimshire, M.J.; Kelly, B.J. Tetrahedron 1989, 45, 2875. (b) Atkinson, R.S.; Jones, D.W.; Kelly, B.J. J. Chem. Soc., Perkin Trans. 1 1991, 1344.

7. Pelter, A.; Ward, R.S. Tetrahedron 2001, 57, 273.

8. For the sulfoximination with LTA see: (a) Anderson, D.J.; Gilchrist, T.L.; Horwell, D.C.; Rees, C.W. J. Chem. Soc., Chem. Commun. 1969, 146. (b) Anderson, D.J.; Horwell, D.C.; Stanton, E.; Gilchrist, T.L.; Rees, C.W. J. Chem. Soc., Perkin Trans. 1 1972, 1317. (c) Colonna, S.; Stirling, C.J.M. J. Chem. Soc., Chem. Commun. 1971, 1591. (d) Colonna, S.; Stirling, C.J.M. J. Chem. Soc., Perkin Trans. 1 1974, 2120.

9. (a) Atkinson, R.S.; Award, S.B. J. Chem. Soc., Perkin Trans. 1 1977, 346. (b) Edwards, M.; Gilchrist, T.L.; Harris, C.J.; Rees, C.W. J. Chem. Res. (Syn.) 1979, 114.

10. Anderson, A. G.Jr.; Fagerburg, D. R Tetrahedron 1973, 2973. (b) Meyer, G. R.; Kellert, C. A.; Ebert R. W. J. Heterocyclic Chem. 1979, 461.

11. Siu, T.; Yudin, A. K. J. Am. Chem. Soc. 2002, 124, 530.

12. Atkinson, R. S.; Malpass, J. R. J. Chem. Soc., Perkin Trans. 1 1977, 2242.

13. Mueller, R. K.; Joos, R.; Felix, D.; Schreiber, J.; Wintner, C.; Eschenmoser, A. Org. Synthesis 1976, 55, 114.

14. Atkinson, R. S.; Anderson, D. J.; Horwell, D. C. J. Chem. Soc. C 1971, 624. 